\title{
Optofluidic Grating Spectrograph on a Chip
}

\author{
Zhenyu Li and Axel Scherer \\ Department of Electrical Engineering, California Institute of Technology, Pasadena, California 91125 \\ zhenyu@caltech.edu, etcher@caltech.edu
}

\begin{abstract}
We demonstrated liquid metal based optical components such as mirrors and reflection gratings for building on-chip optofluidic spectrographs and monochromators. We designed a Czerny-Turner spectrograph with $1.7 \mathrm{~nm}$ resolution, $200 \mathrm{~nm} F S R$ and a footprint of $3 \mathrm{~cm}$ by $3 \mathrm{~cm}$.

(C)2009 Optical Society of America

OCIS codes: (300.6190) Spectrometers; (230.1950) Diffraction gratings; (230.4040) Mirrors; (120.4140) Monochromator; (130.5460) Polymer waveguides; (230.7400) Waveguides, slab; (230.4000) Microstructure fabrication; (130.0130) Integrated optics
\end{abstract}

\section{Introduction}

A central theme of the emerging optofluidic technology is to use liquid-based microfluidic structures to build adaptive and reconfigurable photonic devices [1]. A variety of devices such as microfabricated liquid dye lasers [2], reconfigurable photonic crystal circuits [3] and tunable optical fibers [4] have been demonstrated using liquid gain and dielectric materials. Here we introduce metallic optofluidic components such as on-chip mirrors and reflection gratings made by filling polydimethylsiloxane (PDMS) microfluidic structures with liquid gallium alloy. Such metallic components can be used to build wavefront correction adaptive mirrors, ultrawidely tunable diffraction gratings and on-chip spectrometers.

\section{Liquid Metallic Optofluidic Components}
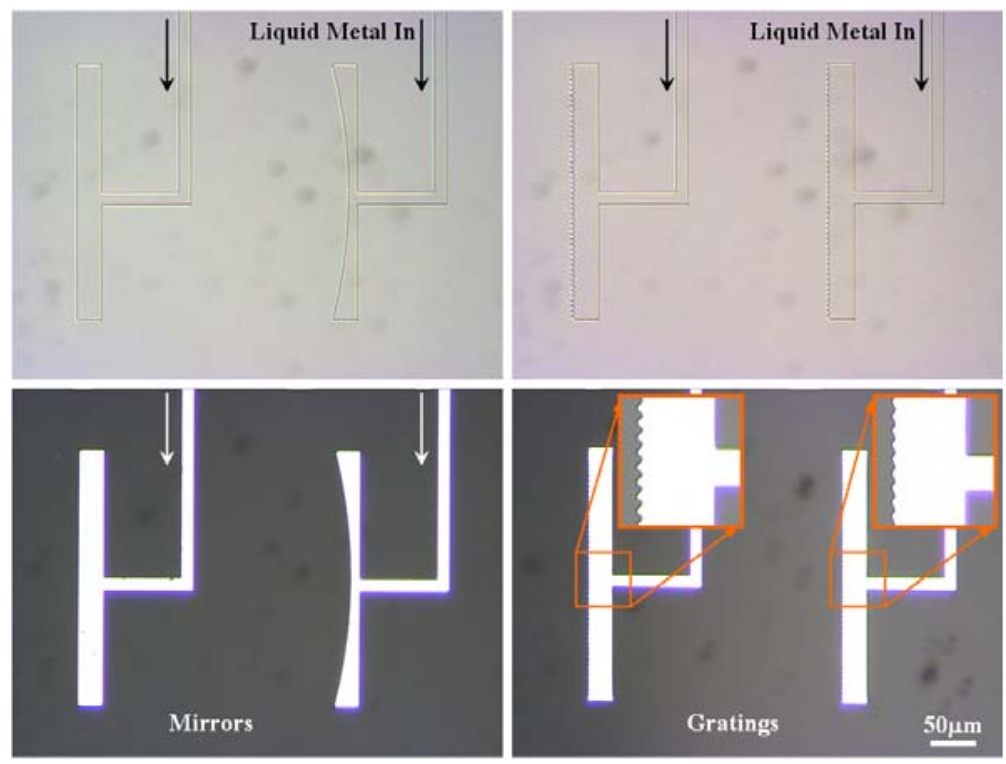

Fig.1. Optical micrographs of on-chip metallic optofluidic mirrors and reflection gratings. Upper: void PDMS microfluidic structures before filled with liquid gallium alloy. Lower: mirrors and gratings formed after filled with liquid gallium alloy. Insets: enlarged regions of gratings. The grating groove density is $200 \mathrm{~g} / \mathrm{mm}$ for both the rectangular and blazed grating. The blaze angle is 30 degree. The radius of curvature of the mirror is $500 \mu \mathrm{m}$.

Figure 1 shows some fabricated metallic optofluidic components on a monolithic PDMS chip using soft lithography as described in Ref $[2,5]$. A patterned microfluidic structure when filled with liquid metal can form mirrors and reflection gratings. We used a gallium-indium-tin eutectic alloy which is liquid at room temperature [6]. Compared to mercury, it's nontoxic, nonevaporative and has higher reflectivity and lower density. So far, we have made both rectangular and blazed gratings with groove density of $200 \mathrm{~g} / \mathrm{mm}$, suitable for high order Echelle spectrometers. With electron beam lithography, we can further increase the groove density to above $5000 \mathrm{~g} / \mathrm{mm}$ which is higher than those of most machine ruled and holographic gratings. As for mirrors, the focal length can be anywhere between a few tens of microns to a few centimeters. An additional advantage of microfabricated mirrors is the ability to fabricate nonspherical shapes which are highly desirable for aberration correction. 


\section{CTuD5.pdf}

\section{Czerny-Turner Spectrograph}

The Czerny-Turner mounting is arguably the most flexible and popular arrangement for grating spectrographs employing plane reflection gratings [7]. In this mounting, as shown in Figure 2, two spherical mirrors are used as collimating and focusing elements. The input slit and spectrum plane are located on either side of the grating.

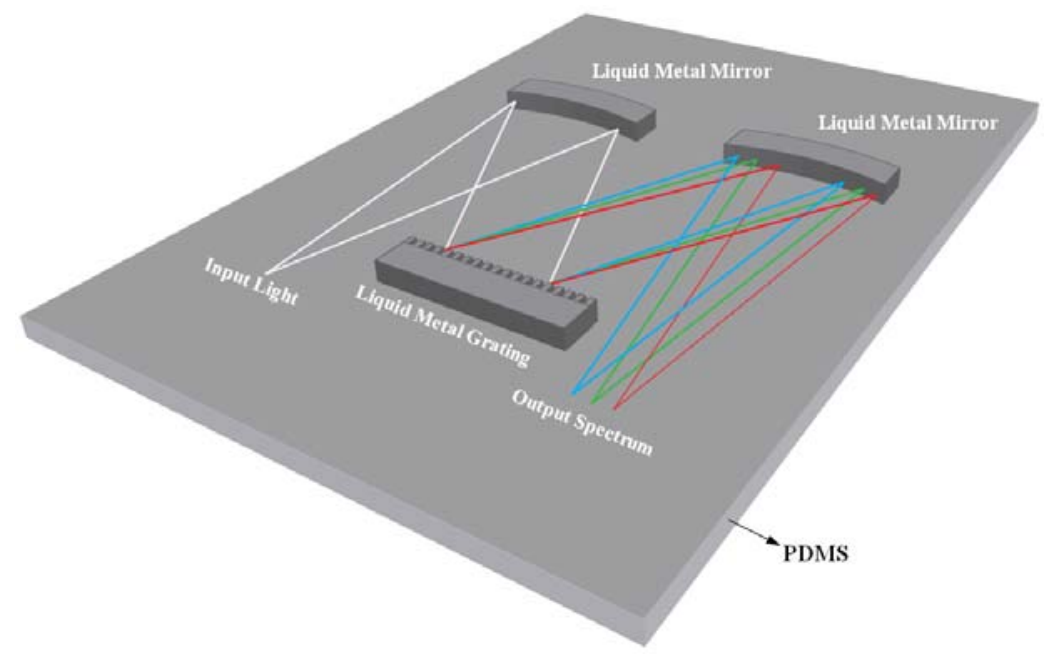

Fig.2. Schematic diagram of a Czerny-Turner optofluidic grating spectrograph. The grating and mirrors are made by filling microfluidic structures with liquid Gallium alloy.

We have designed a symmetric Czerny-Turner spectrograph amenable to on-chip implementation using liquid metallic optical components. The details of the design will be given elsewhere. In terms of performance, the spectrograph has a footprint of $3 \mathrm{~cm}$ by $3 \mathrm{~cm}$, a resolution of $1.7 \mathrm{~nm}$ (resolving power $R \sim 384$ ), a free spectral range (FSR) of $200 \mathrm{~nm}$. The grating has a groove density of $200 \mathrm{~g} / \mathrm{mm}$, a width of $2.5 \mathrm{~mm}$ and is operated in the $3^{\text {rd }}$ order at $600 \mathrm{~nm}$. The incident angle is 7.4 degree. The focal length of the two mirrors is $2.5 \mathrm{~cm}$. We assume the detector pixel size to be 10 micron. The focal number of the resulting system is $\sim \mathrm{F} / 10$. The whole spectrograph is immersed in a two-dimensional slab waveguide with effective index of 1.41. We also designed the spectrograph to satisfy the Rosendahl condition and flat-field condition [7]. The 2D waveguide structure and the small size also contribute to reduce the optical aberrations.

\section{Conclusions}

We have demonstrated liquid gallium alloy based metallic optofluidic components including mirrors and reflection gratings on a PDMS chip. The fabrication and operation of the components is fully compatible with PDMS based microfluidics technology [8]. This opens up the possibility of making on-chip spectrometers integrated with microfluidics.

\section{References}

[1] D. Psaltis, S.R. Quake SR and C.H. Yang, "Developing optofluidic technology through the fusion of microfluidics and optics", Nature, 442 (27), 371-386 (2006).

[2] Z.Y. Li, Z.Y. Zhang, T. Emery, A. Scherer and D. Psaltis, "Single mode optofluidic distributed feedback dye laser", Optics Express, 14(2), 696-701 (2006).

[3] D. Erickson, T. Rockwood, T. Emery, A. Scherer and D. Psaltis, "Nanofluidic tuning of photonic crystal circuits", Optics Letters, 31(1), pp 59-61, (2006).

[4] P. Mach, et al. “Tunable microfluidic optical fiber”. Appl. Phys. Lett. 80, 4294-4296 (2002).

[5] Y.N. Xia and G.M. Whitesides, “Soft lithography,” Annu. Rev. Mater. Sci. 28, 153-184 (1998).

[6] R.N. Lyon Ed. Liquid-Metals Handbook, $2^{\text {nd }}$ edition (Atomic Energy Commission, 1954).

[7] J. James, Spectrograph Design Fundamentals, (Cambridge, 2007), Chap. 8.

[8] M.A. Unger, H.P. Chou, T. Thorsen, A. Scherer and S.R. Quake, "Monolithic microfabricated valves and pumps by multilayer soft lithography," Science, 288, 113-116 (2000).

Acknowledgments

This research was supported by the DARPA center for optofluidic integration and the Boeing Company. 